\title{
Change in energy and entropy of the non-spinning black holes
}

\author{
Dipo Mahto ${ }^{1, a}$, Tanveer Ali Khan ${ }^{2, b}$, Ashok Kumar ${ }^{3, c}$, Raj Kumar Sah ${ }^{4, d}$ \\ ${ }^{1}$ Department of Physics, Marwari College, Tilka Manjhi Bhagalpur University, \\ Bhagalpur - 812007, India \\ ${ }^{2}$ Department of Physics, Tilka Manjhi Bhagalpur University, Bhagalpur - 812007, India \\ ${ }^{3}$ Department of Physics, M. R. J. D. College, Bishnupur, Begusarai - 851101, India \\ ${ }^{4}$ Department of Physics, S. S. V. College, Kahalgoan, Tilka Manjhi Bhagalpur University, \\ Bhagalpur - 812007, India \\ abcdE-mail address: dipomahto@hotmail.com, tanveeralikhan85@gmail.com, \\ drashokbgs259@gmail.com , dr.rajkumarsah@rediffmail.com
}

\begin{abstract}
In the present paper, we have derived an expression for change in entropy of non-spinning black holes on the basis of formula $\left(E_{B H}=K_{B H} R_{S}\right)$ as proposed by Kanak Kumari et al. (2010) and the formula $\delta S_{b h}=\frac{2 \pi}{\kappa \cdot c^{2}} \delta E$ ( Mahto et al., 2011) and calculated their values for different test nonspinning black holes.
\end{abstract}

Keywords: Event-horizon; AGN; Entropy \& Energy

\section{INTRODUCTION}

The laws of black hole mechanics as proposed by Bardeen et al. describe the behaviour of a black hole in close analogy to the laws thermodynamics by relating mass to energy, area to entropy, and surface gravity to temperature [1]. Classically black holes are perfect absorbers, but do not emit anything; their physical temperature is absolute zero [2]. Quantum mechanically, however, there is a possibility that one of a particle production pair in a black hole is able to tunnel the gravitational barrier and escapes the black hole's horizon. Thus, a black hole is not really black; it can radiate or evaporate particles [3]. Dipo Mahto et al. derived an expression for the change in energy and entropy of Non-spinning black holes taking account the first law of the black hole mechanics relating the change in mass $\mathrm{M}$, angular momentum J, horizon area A and charge Q, of a stationary black hole with Einstein's mass-energy equivalence relation [5]. Dipo Mahto et al. justified the validity of the model ( $E_{B H}=K_{B H} R_{s}$ ) as proposed by Kanak Kumari et al. [6]. In the present paper, we have derived an expression for change in entropy of non-spinning black holes using [4] the model ( 
$\left.E_{B H}=K_{B H} R_{s}\right)$ and the formula $\delta S_{b h}=\frac{2 \pi}{\kappa \cdot c^{2}} \delta E[5]$ and calculated their values for different test non-spinning black holes.

\section{DISCUSSION}

The Black hole possesses an event horizon (a one-way membrane) that casually isolates the "inside" of the Black hole from the rest of the universe. The radius of the event horizon of a non-spinning BH given by the Schwarzschild radius in terms of solar mass can be obtained as [7]

$$
R_{s}=2950\left(M / M_{\odot}\right) m
$$

The energy of non-spinning black holes in terms of radius of event horizon is given as [4]

$$
E_{B H}=K_{B H} R_{s}
$$

where

$$
K_{B H}=6.0711 \times 10^{43} \mathrm{~J} / \mathrm{m}
$$

Differentiating eq ${ }^{\mathrm{n}}(2)$, we have

$$
\delta E_{B H}=K_{B H} \delta R_{s}
$$

The change in entropy of different test Non-spinning black holes for their corresponding change in energy is given by the following eqn [5]

$$
\delta S_{b h}=\frac{2 \pi}{\kappa \cdot c^{2}} \delta E
$$

Putting eq ${ }^{\mathrm{n}}$ (4) in the above eqn, we have

$$
\begin{aligned}
& \delta S_{b h}=\frac{2 \pi}{\kappa c^{2}} K_{B H} \delta R_{s} \\
& \delta S_{b h}=\frac{2 \pi}{\kappa c^{2}} K_{B H} \delta R_{b h}
\end{aligned}
$$


where $\kappa$ and $R_{b h}$ are the surface gravity \& radius of the event horizon of black holes given as $[8,10]$

$$
\kappa=\frac{1}{4 M}
$$

The term M stands for the mass of black holes. From eqn. (8), it is clear that the surface gravity of black hole is inversely proportional to its mass and the different black holes will have different surface gravity. The role of surface gravity $(\kappa)$ may be seen in the research paper [8-10].

The equation (7) can be used to calculate the change in entropy of different test Nonspinning black holes for their corresponding change in the radius of the event horizon.

There are two categories of Black holes classified on the basis of their masses clearly very distinct from each other, with very different masses $M \sim 5-20 \mathrm{M}_{\odot}$ for stellar - mass Black holes in X-ray binaries and $\mathrm{M} \sim 10^{6}-10^{9.5} \mathrm{M}_{\odot}$ for super massive black holes in Active Galactic Nuclei $[10,11]$. The other data in the support of mass of black holes in AGN can seen in references [11-16].

On the basis of the data mentioned above regarding the mass of black holes in XRBs and AGN in terms of solar masses, we have calculated energy of black holes and change in entropy in XRBs and AGN for given radius of event horizon of different test Non-spinning black holes listed in the Table 1 and 2 respectively. There is negligible change in the radius of the event horizon. So for numerical calculation, the radius of the event horizon $\left(R_{b h}\right)$ can be used instead of $\delta R_{b h}[5]$.

Table 1. Change in entropy of non-spinning black holes in XRBs.

\begin{tabular}{|c|c|c|c|c|}
\hline S1. No & $\begin{array}{c}\text { Mass of } \\
\text { black holes } \\
(\mathrm{M})\end{array}$ & $\begin{array}{c}\left(\mathrm{R}_{\mathrm{s}}=2950\right. \\
\left.\mathrm{M} / \mathrm{M}_{\odot}\right) \\
(\mathrm{in} \mathrm{metre})\end{array}$ & $\begin{array}{c}\text { Energy of black } \\
\text { holes } \\
\left(\mathrm{E}_{\mathrm{BH}}\right) \text { in Joule. }\end{array}$ & $\begin{array}{c}\text { Entropy change of } \\
\text { Black holes }\left(\delta S_{b h}\right) \\
\text { in J/K }\end{array}$ \\
\hline 1 & $5 \mathrm{M}_{\odot}$ & 14750 & $0.8954 \times 10^{48}$ & $2.483 \times 10^{63}$ \\
\hline 2 & $6 \mathrm{M}_{\odot}$ & 17700 & $1.074 \times 10^{48}$ & $3.575 \times 10^{63}$ \\
\hline 3 & $7 \mathrm{M}_{\odot}$ & 20650 & $1.253 \times 10^{48}$ & $4.867 \times 10^{63}$ \\
\hline 4 & $8 \mathrm{M}_{\odot}$ & 23600 & $1.432 \times 10^{48}$ & $6.357 \times 10^{63}$ \\
\hline 5 & $9 \mathrm{M}_{\odot}$ & 26550 & $1.611 \times 10^{48}$ & $8.045 \times 10^{63}$ \\
\hline 6 & $10 \mathrm{M}_{\odot}$ & 29500 & $1.790 \times 10^{48}$ & $9.932 \times 10^{63}$ \\
\hline 7 & $11 \mathrm{M}_{\odot}$ & 32450 & $1.970 \times 10^{48}$ & $12.018 \times 10^{63}$ \\
\hline 8 & $12 \mathrm{M}_{\odot}$ & 35400 & $2.149 \times 10^{48}$ & $14.303 \times 10^{63}$ \\
\hline 9 & $13 \mathrm{M}_{\odot}$ & 38350 & $2.328 \times 10^{48}$ & $16.786 \times 10^{63}$ \\
\hline 10 & $14 \mathrm{M}_{\odot}$ & 41300 & $2.507 \times 10^{48}$ & $19.468 \times 10^{63}$ \\
\hline
\end{tabular}




\begin{tabular}{|l|l|l|l|l|}
11 & $15 \mathrm{M}_{\odot}$ & 44250 & $2.686 \times 10^{48}$ & $22.349 \times 10^{63}$ \\
\hline 12 & $16 \mathrm{M}_{\odot}$ & 47200 & $2.865 \times 10^{48}$ & $25.428 \times 10^{63}$ \\
\hline 13 & $17 \mathrm{M}_{\odot}$ & 50150 & $3.044 \times 10^{48}$ & $28.706 \times 10^{63}$ \\
\hline 14 & $18 \mathrm{M}_{\odot}$ & 53100 & $3.223 \times 10^{48}$ & $32.182 \times 10^{63}$ \\
\hline 15 & $19 \mathrm{M}_{\odot}$ & 56050 & $3.402 \times 10^{48}$ & $35.857 \times 10^{63}$ \\
\hline 16 & $20 \mathrm{M}_{\odot}$ & 59000 & $3.581 \times 10^{48}$ & $39.731 \times 10^{63}$ \\
\hline
\end{tabular}

Table 2. Energy and change in entropy of Non-spinning Black holes in AGN.

\begin{tabular}{|c|c|c|c|c|c|c|c|}
\hline $\begin{array}{l}\text { Sl. } \\
\text { No. }\end{array}$ & $\begin{array}{l}\text { Mass of BHs } \\
\text { (M) }\end{array}$ & $\begin{array}{c}\left(\mathrm{R}_{\mathrm{bh}}=2950\right. \\
\left.\mathrm{M} / \mathrm{M}_{\odot}\right) \\
\text { (in metre) }\end{array}$ & $\begin{array}{c}\log \left(R_{\mathrm{bh}}\right) \\
\text { (in metre) }\end{array}$ & $\begin{array}{c}\mathrm{E}_{\mathrm{BH}} \text { (in } \\
\text { Joule) }\end{array}$ & $\log \left(E_{\mathrm{BH}}\right)$ & $\begin{array}{l}\text { Entropy change } \\
\text { of Black holes } \\
\left(\delta S_{b h}\right) \text { in } \mathrm{J} / \mathrm{K}\end{array}$ & $\begin{array}{l}\log ( \\
\left.\delta S_{b h}\right)\end{array}$ \\
\hline 1 & $1 \times 10^{6} \mathrm{M}_{\odot}$ & $2.950 \times 10^{9}$ & 9.4698 & $1.790 \times 10^{53}$ & 53.2529 & $9.932 \times 10^{73}$ & 73.9970 \\
\hline 2 & $2 \times 10^{6} \mathrm{M}_{\odot}$ & $5.950 \times 10^{9}$ & 9.7709 & $3.612 \times 10^{53}$ & 53.5565 & $39.731 \times 10^{73}$ & 74.5991 \\
\hline 3 & $3 \times 10^{6} \mathrm{M}_{\odot}$ & $8.850 \times 10^{9}$ & 9.9469 & $5.372 \times 10^{53}$ & 53.7302 & $89.396 \times 10^{73}$ & 74.9513 \\
\hline 4 & $4 \times 10^{6} \mathrm{M}_{\odot}$ & $1.180 \times 10^{10}$ & 10.0719 & $7.163 \times 10^{53}$ & 53.8551 & $15.892 \times 10^{74}$ & 75.2012 \\
\hline 5 & $5 \times 10^{6} \mathrm{M}_{\odot}$ & $1.475 \times 10^{10}$ & 10.1688 & $8.954 \times 10^{53}$ & 53.9520 & $24.832 \times 10^{74}$ & 75.3948 \\
\hline 6 & $6 \times 10^{6} \mathrm{M}_{\odot}$ & $1.770 \times 10^{10}$ & 10.2480 & $1.074 \times 10^{54}$ & 54.0310 & $35.758 \times 10^{74}$ & 75.5533 \\
\hline 7 & $7 \times 10^{6} \mathrm{M}_{\odot}$ & $2.065 \times 10^{10}$ & 10.3149 & $1.253 \times 10^{54}$ & 54.0979 & $48.671 \times 10^{74}$ & 75.6872 \\
\hline 8 & $8 \times 10^{6} \mathrm{M}_{\odot}$ & $2.360 \times 10^{10}$ & 10.3729 & $1.432 \times 10^{54}$ & 54.1559 & $63.570 \times 10^{74}$ & 75.8033 \\
\hline 9 & $9 \times 10^{6} \mathrm{M}_{\odot}$ & $2.655 \times 10^{10}$ & 10.4241 & $1.611 \times 10^{54}$ & 54.2071 & $80.456 \times 10^{74}$ & 75.9056 \\
\hline 10 & $1 \times 10^{7} \mathrm{M}_{\odot}$ & $2.950 \times 10^{10}$ & 10.4698 & $1.790 \times 10^{54}$ & 54.2529 & $9.932 \times 10^{75}$ & 75.9970 \\
\hline 11 & $2 \times 10^{7} \mathrm{M}_{\odot}$ & $5.950 \times 10^{10}$ & 10.7709 & $3.612 \times 10^{54}$ & 54.5565 & $39.731 \times 10^{75}$ & 76.5991 \\
\hline 12 & $3 \times 10^{7} \mathrm{M}_{\odot}$ & $8.850 \times 10^{10}$ & 10.9469 & $5.372 \times 10^{54}$ & 54.7302 & $89.396 \times 10^{75}$ & 76.9513 \\
\hline 13 & $4 \times 10^{7} \mathrm{M}_{\odot}$ & $1.180 \times 10^{11}$ & 11.0719 & $7.163 \times 10^{54}$ & 54.8551 & $15.892 \times 10^{76}$ & 77.2012 \\
\hline 14 & $5 \times 10^{7} \mathrm{M}_{\odot}$ & $1.475 \times 10^{11}$ & 11.1688 & $8.954 \times 10^{54}$ & 54.9520 & $24.832 \times 10^{76}$ & 77.3948 \\
\hline 15 & $6 \times 10^{7} \mathrm{M}_{\odot}$ & $1.770 \times 10^{11}$ & 11.2480 & $1.074 \times 10^{55}$ & 55.0310 & $35.758 \times 10^{76}$ & 77.5533 \\
\hline
\end{tabular}




\begin{tabular}{|c|c|c|c|c|c|c|c|}
\hline 16 & $7 \times 10^{7} \mathrm{M}_{\odot}$ & $2.065 \times 10^{11}$ & 11.3149 & $1.253 \times 10^{55}$ & 55.0979 & $48.671 \times 10^{76}$ & 77.6872 \\
\hline 17 & $8 \times 10^{7} \mathrm{M}_{\odot}$ & $2.360 \times 10^{11}$ & 11.3729 & $1.432 \times 10^{55}$ & 55.1559 & $63.570 \times 10^{76}$ & 77.8033 \\
\hline 18 & $9 \times 10^{7} \mathrm{M}_{\odot}$ & $2.655 \times 10^{11}$ & 11.4241 & $1.611 \times 10^{55}$ & 55.2071 & $80.456 \times 10^{76}$ & 77.9056 \\
\hline 19 & $1 \times 10^{8} \mathrm{M}_{\odot}$ & $2.950 \times 10^{11}$ & 11.4698 & $1.790 \times 10^{55}$ & 55.2529 & $9.932 \times 10^{77}$ & 77.9970 \\
\hline 20 & $2 \times 10^{8} \mathrm{M}_{\odot}$ & $5.950 \times 10^{11}$ & 11.7709 & $3.162 \times 10^{55}$ & 55.5565 & $39.731 \times 10^{77}$ & 78.5991 \\
\hline 21 & $3 \times 10^{8} \mathrm{M}_{\odot}$ & $8.850 \times 10^{11}$ & 11.9469 & $5.372 \times 10^{55}$ & 55.7302 & $89.396 \times 10^{77}$ & 78.9513 \\
\hline 22 & $4 \times 10^{8} \mathrm{M}_{\odot}$ & $1.180 \times 10^{12}$ & 12.0719 & $7.163 \times 10^{55}$ & 55.8551 & $15.892 \times 10^{78}$ & 79.2012 \\
\hline 23 & $5 \times 10^{8} \mathrm{M}_{\odot}$ & $1.475 \times 10^{12}$ & 12.1688 & $8.954 \times 10^{55}$ & 55.9520 & $24.832 \times 10^{78}$ & 79.3948 \\
\hline 24 & $6 \times 10^{8} \mathrm{M}_{\odot}$ & $1.770 \times 10^{12}$ & 12.2480 & $1.074 \times 10^{56}$ & 56.0310 & $35.758 \times 10^{78}$ & 79.5533 \\
\hline 25 & $7 \times 10^{8} \mathrm{M}_{\odot}$ & $2.065 \times 10^{12}$ & 12.3149 & $1.253 \times 10^{56}$ & 56.0979 & $48.671 \times 10^{78}$ & 79.6872 \\
\hline 26 & $8 \times 10^{8} \mathrm{M}_{\odot}$ & $2.360 \times 10^{12}$ & 12.3729 & $1.432 \times 10^{56}$ & 56.1559 & $63.570 \times 10^{78}$ & 79.8033 \\
\hline 27 & $9 \times 10^{8} \mathrm{M}_{\odot}$ & $2.655 \times 10^{12}$ & 12.4241 & $1.611 \times 10^{56}$ & 56.2071 & $80.456 \times 10^{78}$ & 79.9056 \\
\hline 28 & $1 \times 10^{9} \mathrm{M}_{\odot}$ & $2.950 \times 10^{12}$ & 12.4698 & $1.790 \times 10^{56}$ & 56.2529 & $9.932 \times 10^{79}$ & 79.9970 \\
\hline 29 & $2 \times 10^{9} \mathrm{M}_{\odot}$ & $5.950 \times 10^{12}$ & 12.7709 & $3.612 \times 10^{56}$ & 56.5565 & $39.731 \times 10^{79}$ & 80.5991 \\
\hline 30 & $3 \times 10^{9} \mathrm{M}_{\odot}$ & $8.850 \times 10^{12}$ & 12.9469 & $5.372 \times 10^{56}$ & 56.7302 & $89.396 \times 10^{79}$ & 80.9513 \\
\hline 31 & $4 \times 10^{9} \mathrm{M}_{\odot}$ & $1.180 \times 10^{13}$ & 13.0719 & $7.163 \times 10^{56}$ & 56.8551 & $15.892 \times 10^{80}$ & 81.2012 \\
\hline 32 & $5 \times 10^{9} \mathrm{M}_{\odot}$ & $1.475 \times 10^{13}$ & 13.1688 & $8.954 \times 10^{56}$ & 56.9520 & $28.432 \times 10^{80}$ & 81.3948 \\
\hline 33 & $6 \times 10^{9} \mathrm{M}_{\odot}$ & $1.770 \times 10^{13}$ & 13.2480 & $1.074 \times 10^{57}$ & 57.0310 & $35.758 \times 10^{80}$ & 81.5533 \\
\hline 34 & $7 \times 10^{9} \mathrm{M}_{\odot}$ & $2.065 \times 10^{13}$ & 13.3149 & $1.253 \times 10^{57}$ & 57.0979 & $48.671 \times 10^{80}$ & 81.6872 \\
\hline 35 & $8 \times 10^{9} \mathrm{M}_{\odot}$ & $2.360 \times 10^{13}$ & 13.3729 & $1.432 \times 10^{57}$ & 57.1559 & $63.570 \times 10^{80}$ & 81.8033 \\
\hline 36 & $9 \times 10^{9} \mathrm{M}_{\odot}$ & $2.655 \times 10^{13}$ & 13.4241 & $1.611 \times 10^{57}$ & 57.2071 & $80.456 \times 10^{80}$ & 81.9056 \\
\hline 37 & $1 \times 10^{10} \mathrm{M}_{\odot}$ & $2.950 \times 10^{13}$ & 13.4698 & $1.790 \times 10^{57}$ & 57.2529 & $9.932 \times 10^{81}$ & 81.9970 \\
\hline
\end{tabular}




\section{Graph 1:}

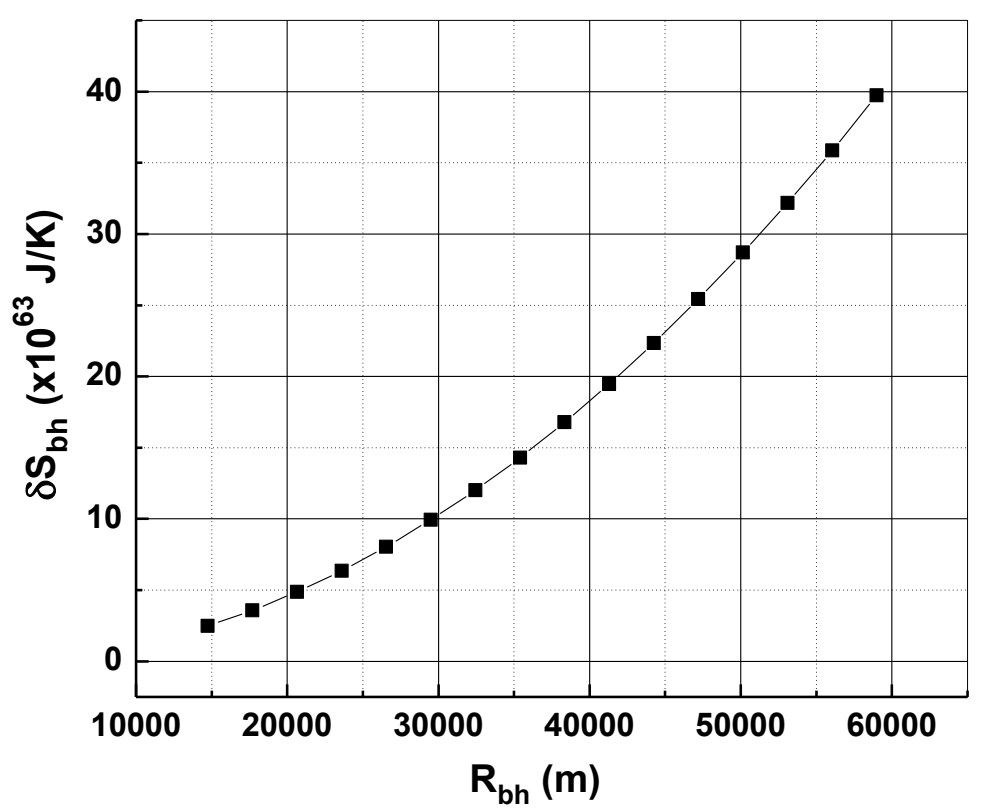

Fig. 3. The graph plotted between the radius of event horizon and corresponding change in entropy of the black hole in XRBs (Table 1).

\section{Graph 2:}

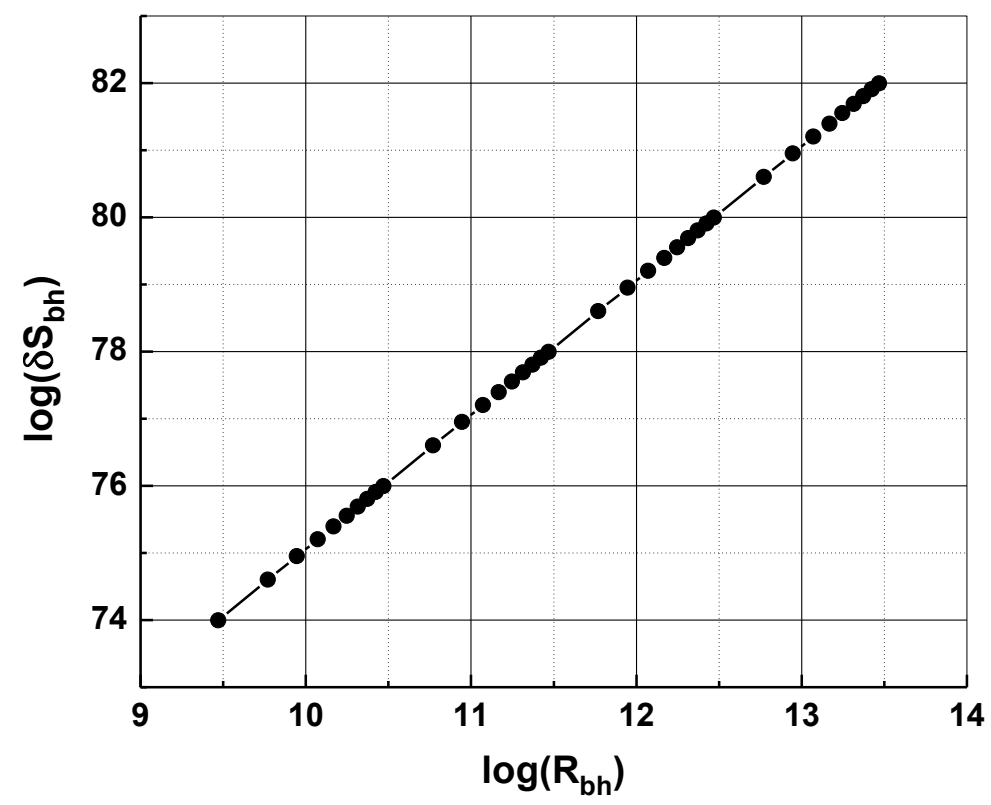

Fig. 4. The graph plotted between the radius of event horizon and corresponding change in entropy of the black hole in AGN (Table 2). 


\section{DISCUSSION AND RESULT}

In present work, we have derived the formula for the change in entropy of non-spinning black holes represented by $\delta S_{b h}=\frac{2 \pi}{\kappa c^{2}} K_{B H} \delta R_{b h}$ using $E_{B H}=K_{B H} R_{s}$ in equation for change in entropy $\delta S_{b h}=\frac{2 \pi}{\kappa \cdot c^{2}} \delta E$. We have calculated the change in entropy of different test non spinning black holes in X-ray binaries (XRBs) and Active Galactic Nuclei (AGN) and the graphs have been plotted between:

(A) the radius of event horizon $\left(\mathrm{R}_{\mathrm{bh}}\right)$ of different test black holes and their corresponding change in entropy in XRBs (Fig. 1).

(B) the radius of event horizon $\left(\mathrm{R}_{\mathrm{bh}}\right)$ of different test black holes and their corresponding change in entropy in AGN (Fig. 2).

From the observation of the data in the Table 1 and 2, it is clear that the change in entropy of different test black holes increases with increase in corresponding values of the mass/radius of the event horizon in both case of XRBs and AGN. From the observation of the graph in the Figure 1, it is clear that the change in entropy of different test black holes increases approximately linearly (slightly curved) with increase in corresponding values of the mass/radius of the event horizon in the case of XRBs, while linearly in the case of AGN.

The graph plotted between the radius of event horizon $\left(\mathrm{R}_{\mathrm{bh}}\right)$ of different test black holes $\&$ their corresponding change in entropy in XRBs (Fig. 1) has the same nature of the graph plotted between the radius of event horizon $\left(\mathrm{R}_{\mathrm{bh}}\right)$ of different test black holes and their corresponding entropy in XRBs using the well known relation of entropy $S=A / 4=\pi R_{b h}^{2} / 4$ as proposed by Stephen Hawking (Hawking 1973, Mahto et al., 2012) and the graph plotted between the radius of event horizon $\left(\mathrm{R}_{\mathrm{bh}}\right)$ of different test black holes and their corresponding change in entropy in AGN (Fig. 2) is straight line justifying the validity of $E_{B H}=K_{B H} R_{S}$ as proposed by Kanak et al., (2010), because the relation $\delta S_{b h}=\frac{2 \pi}{\kappa c^{2}} K_{B H} \delta R_{b h}$ has been derived using relation $E_{B H}=K_{B H} R_{s}$.

\section{CONCLUSIONS}

In the present research paper, we have drawn the following conclusions:

A) The calculated values for change in entropy show that $\delta S \geq 0$ for each black hole candidates existing in XRBs and AGN. This result is good agreement with the second law of thermodynamics.

B) Larger the mass/ radius of event horizon, greater is the change in entropy of black holes and vice-versa.

C) Our result has good agreement in XRBs with relation of entropy $S=A / 4=\pi R_{b h}^{2} / 4$ as proposed by Hawking.

D) Kanak model for energy of the non-spinning black hole is justified. 


\section{Acknowledgement}

The authors are grateful to the referee for pointing out the errors in the original manuscript and making constructive suggestions. The authors also acknowledge the help of Dr. D. T. K. Dutta, Retired Professor, University Department of English, T. M. B. U., Bhagalpur and Prof. Vijoy Kant Mishra, H.O.D. Physics, Marwari College, Bhagalpur in proof reading. The authors are obliged to Dr. Gopi Kant Jha, Former Head \& Prof. of Physics, L.N.M.U Darbhanga and Dr. Neeraj Pant, Associate Professor, Dept. of Mathematics, N.D.A. Khadakwasala, Pune for their inspiration and motivation.

\section{Reference}

[1] Bardeen J. M., Carter B., Hawking S. W., Commun. Math. Phy. 31(2) (1973) 161-170.

[2] Wald R. M. (2001). The thermodynamics of black holes. Living reviews in relativity. www.livingreviews.org \article \volumel2001-6-wald

[3] A. Triyanta, N. Bowaire, J. Math. Fund. Sci. 45(2) (2013) 114-123.

[4] Kumari K., Mahto D., Chandra G., Sah R. K., Singh K. M., Bulletin of Pure and Applied Sciences 29D(2)P (2010) 183-187.

[5] Mahto D., Kumari K., Sah R. K., Singh K. M.: "Study of Non-spinning black holes with reference to the change in Energy and Entropy". Astrophys Space Sci.

Doi 10.1007/s10509-011-0883-7(2011).

[6] Mahto D., Mehta R. N., Parhi K., Singh K. M., Sah R. K., SJPS 3(2) (2013) 1-5. http://www.sjps.org/ (C) American V-King Scientific Publishing.

[7] Einstein A., "On a stationary system with spherical symmetry consisting of many gravitating masses". Annals of Mathematics (1939).

[8] Transchen J., An introduction to black hole evaporation, General Relativity and Quantum Cosmology (2000). http://arxiv.org/abs/gr-qc/0010055v1

[9] Dabholkar A., Curr. Sci. 89(12) (2005) 25.

[10] Mahto D., Prakash V., Prasad U., Singh B. K., Singh K. M., "Change in entropy of nonspinning black holes w.r,t. the radius of event horizon in XRBs". Astrophys Space Sci. Doi 10.1007/s10509-012-1219-y(2012).

[11] R. Narayan, Black Holes In Astrophysics, New Journal Physics 7(1) (2005)1-31.

[12] D. Mahto, M. S. Nadeem, M. Ram, K. Vineeta, Journal of Gravity 2013 (2013), Article ID: 222676. http://dx.doi.org/10.1155/2013/232676

[13] Ferrarese L., Ford H., Space Science Reviews 116 (2005) 523-624.

[14] Grey Madejski, Black Holes in Active Galactic Nuclei, SLAC-PUB-9702,March 2003.

[15] R. D. Blandford, Astrophysics and Space Science 261 (1999) 245-252.

[16] W. L. W. Sargent, et al., The Astrophysical Journal 221 (1978) 731. 